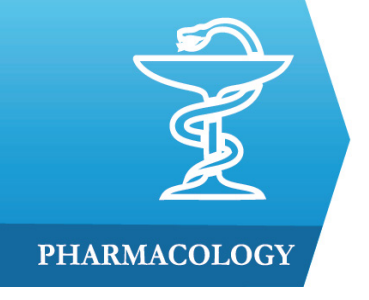

1) Physiology Department, Functional Biosciences Division, Iuliu Hatieganu University of Medicine and Pharmacy, Cluj-Napoca, Romania

2) Pathophysiology Department. Functional Biosciences Division, Iuliu Hatieganu University of Medicine and Pharmacy, Cluj-Napoca, Romania

3) Internal Medicine Department, Iuliu Hatieganu University of Medicine and Pharmacy, Cluj-Napoca, Romania

DOI: $10.15386 / \mathrm{mpr}-1484$

Manuscript received: 07.10.2019

Received in revised form: 20.12.2019

Accepted: 15.01.2020

Address for correspondence:

rdmitrea@yahoo.co.uk

This work is licensed under a Creative Commons Attribution-NonCommercialNoDerivatives 4.0 International License

\title{
Quercetin and curcumin effects in experimental pleural inflammation
}

Cristina Bidian ${ }^{1}$, Daniela-Rodica Mitrea ${ }^{1}$, Olivia Gabriela Vasile ${ }^{1}$, Adriana Filip ${ }^{1}$, Adriana Florinela Cătoi ${ }^{2}$, Remus Moldovan ${ }^{1}$, Nicoleta Decea ${ }^{1}$, Adriana $\mathrm{Albu}^{3}$

\begin{abstract}
Background. The inflammatory mechanisms occur with the highest prevalence in pulmonary pathology in addition to oxidative stress and activation of intracellular signaling pathways. The oxidative stress represents the imbalance between pro-oxidants and antioxidants which can lead to the activation of the oxidative mechanisms with noxious potential to the body. Therefore, finding a therapy that would counteract the injurious effects of free radicals and inflammation is highly attractive. Quercetin is the most active flavonoid, with important anti-inflammatory and antioxidant effects, while curcumin has antioxidant effects that are similar to the standard antioxidants and exerts direct anti-inflammatory activity.
\end{abstract}

Aims. The aim of this study is to evaluate the antioxidant effects of quercetin and curcumin on an experimental model, pleural inflammation induced by carrageenan.

Methods. Eight groups of adult male rats were used: Ia and Ib - control groups, IIa and IIb - with carrageenan administration, IIIa and IIIb - received curcumin and carrageenan, IVa and IVb - quercetin and carrageenan administration. Blood and lung samples were taken at 4 hours (Ia, IIa, IIIa, IVa groups) and at 24 hours (Ib, IIb, IIIb, IVb groups) after carrageenan injection.

Results. At 4 and at 24 hours, curcumin and quercetin have shown protective systemic effects, decreasing significantly the oxidative stress (malondialdehyde level) and stimulating significantly the antioxidant protection (ceruloplasmin and glutathione levels) compared to the group that received only carrageenan. In the lungs, at 4 hours, the redox misbalance was significantly reduced only in animals that were treated with quercetin, modifications that were not observed at 24 hours.

Conclusions. In serum, curcumin presented higher antioxidant effects, compared to quercetin. In lungs, quercetin administration showed superior beneficial effects, but only temporarily.

Keywords: pleural inflammation, oxidative stress, carrageenan, curcumin, quercetin

\section{Background and aims}

Pulmonary pathology includes a largerange ofdisorders, with inflammatory diseases (bronchitis, asthma) among the first. Inflammation represents all the secondary tissue alterations that occur during physical, chemical, mechanical or infectious injuries. During these processes, high amounts of diverse active molecules are released from the wounded tissue area, including reactive oxygen species, cytokines, adhesion molecules, transcription factors, enzymes etc.

The oxidative stress represents the imbalance between pro-oxidants and antioxidants towards the oxidative processes with destructive potential. Considering either the defense reactions or the degenerative processes, the body is continuously exposed to the oxidative stress. The antioxidants are endogenous or exogenous compounds that protect the biological systems from the noxious action of the excessive oxidation products. 
Their mechanism of action may involve various processes: inhibition of the enzymatic reactions, blockage of the inflammatory mediators' production or products depuration. Therefore, finding a therapy that would counteract the injurious effects of free radicals and inflammation and protect the cells is highly attractive.

Flavonoids represent a group of pigments extracted from plants, responsible for the flower and fruit colors. Over 4000 flavonoids have been described and characterized by their chemical structure. A high amount of flavonoids has been found in citrus fruit, onion, parsley, berries, green tea and red wine. Flavonoids are known for their anti-inflammatory, antioxidant, anti-allergic, antiviral, and anticancer properties [1]. In experimental studies, quercetin has been identified as the most active flavonoid. After absorption, quercetin is conjugated in the liver with glucuronic acid. In the inflamed area, neutrophils release $\beta$-glucuronidase that deconjugates quercetin, making it active, a mechanism demonstrated by in vitro studies [2]. The anti-inflammatory and antioxidant effects were due to the direct inhibition of the early processes of the inflammation, through the interaction with the calcium channels and/or calmodulin, blocking in this way the first messenger of the intercellular signalling. In addition, quercetin can modulate the antioxidant activity of vitamin $C[3,4]$.

Curcumin, a biphenolic natural compound, part of the curcuminoids class, is a yellow pigment obtained from turmeric, the root of Curcuma longa. The curcumin antioxidant effect is similar to that of the standard antioxidants, like vitamin $\mathrm{C}$ and vitamin $\mathrm{E}$ [5]. In experimental models of acute inflammation, curcumin has shown comparable effect to those of cortisone or phenylbutazone, but in chronic inflammations the effect was halved [6]. Curcumin exerted direct anti-inflammatory actions through different mechanisms: inhibition of leukotrienes synthesis or platelet aggregation, stimulation of fibrinolysis, inhibition of neutrophils response to various stimuli and induced lysosomal membrane stabilization. In addition, curcumin modulated the corticosteroids release from the suprarenal glands, improved the cortisone effects through receptors sensitisation, and increased the half-life of endogenous cortisone by altering its hepatic degradation.

The present study investigated the effects of quercetin and curcumin administration in an experimental model of carrageenan-induced pleural inflammation in Wistar rats. Carrageenan is a generic name given to the family of natural carbohydrates obtained through extraction from different types of seaweed. It does not have nutritional value but is used in food industry, for its properties (emulsifiability, viscosity), and in pharmaceutical industry to test the activity of anti-inflammatory agents. Carrageenan-induced inflammation is the most popular, highly sensitive and reproducible test used for testing of nonsteroidal antiinflammatory agents and has been established as a valid model to study new anti-inflammatory drugs [7].
The aim of this study was to analyze and quantify the local (in lung homogenates) and general (in serum) antioxidant effects of curcumin and quercetin.

\section{Methods}

\section{Chemicals}

All reagents were purchased from Sigma-Aldrich Germany.

Fifty $\mathrm{mg} / \mathrm{kg} /$ day of curcumin or quercetin were administered dissolved in $0.5 \mathrm{ml}$ vehicle CMC $0.5 \%$ $(0.5 \mathrm{~g}$ carboxymethyl cellulose dissolved in $100 \mathrm{ml}$ physiological salt).

\section{Experimental animals}

The experiments were performed in the Experimental Research Laboratory of the Physiology Department, Iuliu Haţieganu University of Medicine and Pharmacy, ClujNapoca. Adult male rats, Wistar breed, with weights between 200-220 g, supplied by the University Biobase were placed in cages and maintained in the same room, at $21^{\circ} \mathrm{C}$ degrees, with $12 \mathrm{~h}$ light $/ 12 \mathrm{~h}$ dark cycle, with access to food and water ad libitum. The tests were conducted under the guidelines of the Directive 89/609/EEC and of the Ethical Committee of Iuliu Haţieganu University of Medicine and Pharmacy, Cluj-Napoca (nr. 229/12.06.2014).

\section{Experimental design}

The animals were randomly allocated into 8 groups, with 8 animals in each group and received, for 4 days, at 7 a.m., $0.5 \mathrm{ml}$ of vehicle or $0.5 \mathrm{ml}$ of antioxidant solution, through oral gavage. On the fifth day, $0.2 \mathrm{ml}$ of physiological salt or carrageenan $1 \%$ was intrapleurally administered. The experimental groups were the following:

$I a$ and Ib (control groups): $0.5 \mathrm{ml}$ vehicle through oral gavage for four days, and on the fifth day $0.2 \mathrm{ml}$ of physiological salt in intrapleural injection;

IIa and IIb groups: $0.5 \mathrm{ml}$ vehicle through oral gavage for four days, and on the fifth day $0.2 \mathrm{ml}$ of carrageenan $1 \%$, in intrapleural injection;

III $a$ and IIIb: $50 \mathrm{mg} / \mathrm{kg} / \mathrm{day}$ curcumin dissolved in $0.5 \mathrm{ml}$ vehicle $\mathrm{CMC} 0.5 \%$ through oral gavage for four days, and on the fifth day $0.2 \mathrm{ml}$ of carrageenan $1 \%$, in intrapleural injection;

$I V a$ and $I V b: 50 \mathrm{mg} / \mathrm{kg} /$ day quercetin dissolved in $0.5 \mathrm{ml}$ vehicle CMC $0.5 \%$ through oral gavage for four days, and on the fifth day $0.2 \mathrm{ml}$ of carrageenan $1 \%$, in intrapleural injection.

Venous blood was collected from retro-orbital sinus and lung fragments (through thoracotomy) at 4 hours (Ia, IIa, IIIa and IVa groups) and at 24 hours after carrageenan administration (Ib, IIb, IIIb and IVb groups).

The experiments were performed under general anesthesia with ketamine $10 \%$ and xylazine $2 \%$ administered by intramuscular injection. An incision through the sixth intercostal space was performed, subjacent musculature was sectioned and inside the pleural cavity were injected 
$0.2 \mathrm{ml}$ of carrageenan $1 \%$. The incision was then closed (adapted technique after Petronilho et al. 2010) [8].

Biochemical determinations were performed in the Laboratory of Research in Oxidative Stress, Physiology Discipline, Iuliu Haţieganu University of Medicine and Pharmacy Cluj-Napoca. The parameters of the pro-oxidant/ antioxidant balance were evaluated in the serum and pulmonary tissue homogenates: malondialdehyde (MDA) (Conti et al. technique 1991) [9], glutathione (GSH) (Hu method, 1994) [10] and ceruloplasmine (Cp) (Ravin method, 1961) [11].

\section{Data analysis}

The obtained values were statistically processed using GraphPad Prism version 5.03 for Windows, GraphPad Software, (San Diego California USA), performing One-way ANOVA followed by the Post-test Bonfferoni. The threshold significance level was $\mathrm{p}<0.05(* * * \mathrm{p}<0.001$; $\left.* * \mathrm{p}<0.01 ;{ }^{*} \mathrm{p}<0.05\right)$.

\section{Results}

a) Quercetin and curcumin effects on systemic oxidative stress parameters at 4 hours after inflammation induction

In serum, malondialdehyde increased significantly in rats with carrageenan administration (group IIa), compared to control group (Ia, $\mathrm{p}<0.05$ ) and to treated groups (IIIa and IVa, $\mathrm{p}<0.01$ ) (Figure 1).

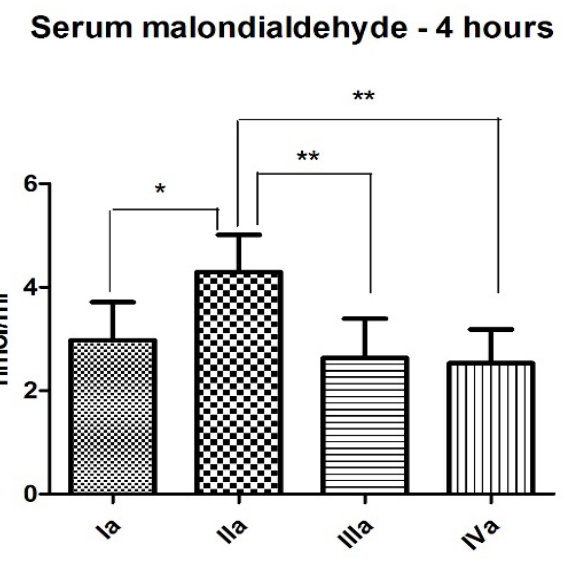

Figure 1. Malondialdehyde levels in serum, in groups pretreated with quercetin and curcumin at 4 hours after carrageenan administration.

Ceruloplasmin concentration was significantly increased in rats that were treated with antioxidants (IIIa and IVa groups), in comparison with the animals that did not receive medication (groups Ia and IIa, $\mathrm{p}<0.001$ ). Curcumin stimulated the synthesis of ceruloplasmin in the liver (group IIIa), compared to quercetin group (IVa, $\mathrm{p}<0.001$ ) (Figure 2).

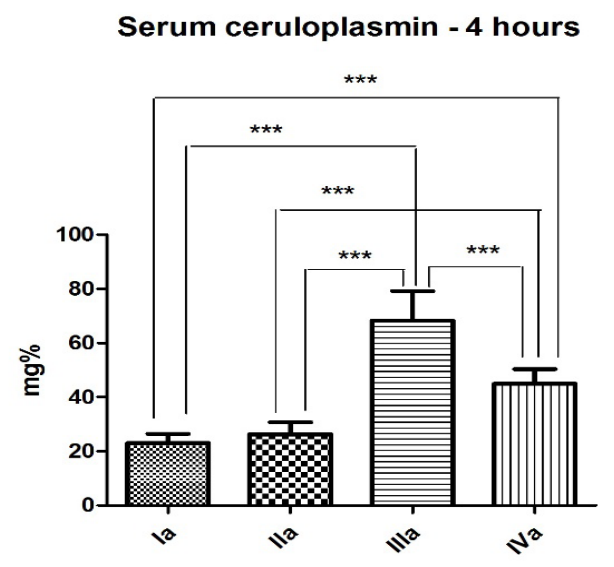

Figure 2. Ceruloplasmin levels in serum, in animals pretreated with quercetin and curcumin at 4 hours after carrageenan administration.

Glutathione levels in serum increased significantly in groups that received antioxidants (IIIa and IVa, $\mathrm{p}<0.001$ ), compared to the carrageenan group (IIa). Curcumin administration (group IIIa) increased significantly the glutathione concentration, in comparison to control group (Ia, p <0.05). Carrageenan administration (group IIa) reduced significantly the level of glutathione in serum, compared to the control group (Ia, $\mathrm{p}<0.05$ ) (Figure 3).

\section{Serum glutathione -4 hours}

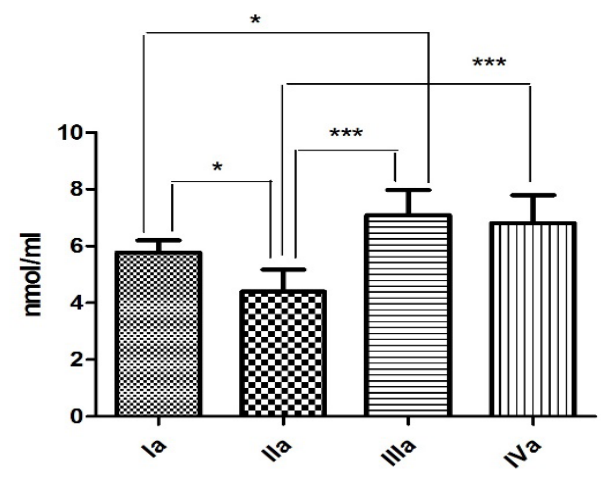

Figure 3. Glutathione levels in serum, in animals pretreated with quercetin and curcumin at 4 hours after carrageenan administration.

b) Quercetin and curcumin effects on systemic oxidative stress parameters at 24 hours after inflammation induction

In serum, at 24 hours after intrapleural carrageenan administration, malondialdehyde presented significant decreases in the groups that were previously treated with antioxidants (groups IIIb, $\mathrm{p}<0.01$ and IVb, $\mathrm{p}<0.001$ ), in comparison with the control (Ib) and carrageenan (IIb) groups (Figure 4). 
Serum malondialdehyde - 24 hours

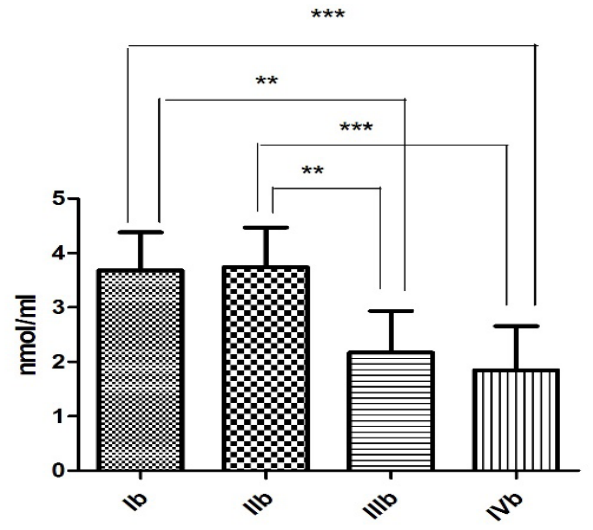

Figure 4. Malondialdehyde levels in serum, in animals pretreated with quercetin and curcumin at 24 hours after carrageenan administration.

Ceruloplasmin presented significant increases in groups that received antioxidants (groups IIIb and IVb), in comparison with both untreated groups (control group $\mathrm{Ib}$ and carrageenan group IIb, $\mathrm{p}<0.001)$. Significant differences were recorded also between the groups that received antioxidants, with significant higher values in rats that received quercetin (group IVb, $\mathrm{p}<0.001$ ) (Figure 5).

\section{Serum ceruloplasmin - 24 hours}

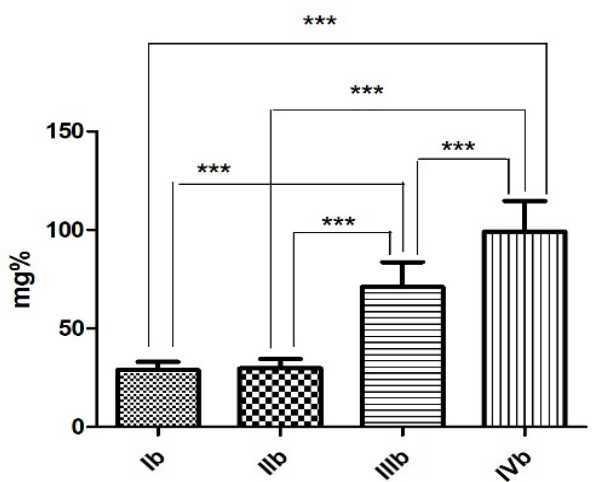

Figure 5. Ceruloplasmin levels in the serum of animals pretreated with quercetin and curcumin, at 24 hours after carrageenan administration.

In serum, at 24 hours after carrageenan administration, compared to the untreated groups (control Ib and carrageenan $\mathrm{IIb}$ ), the reduced glutathione increased significantly in rats that received antioxidants, with the highest values in curcumin group (IIIb, $\mathrm{p}<0.001$ ) (Figure 6).

\section{Serum glutathione - 24 hours}

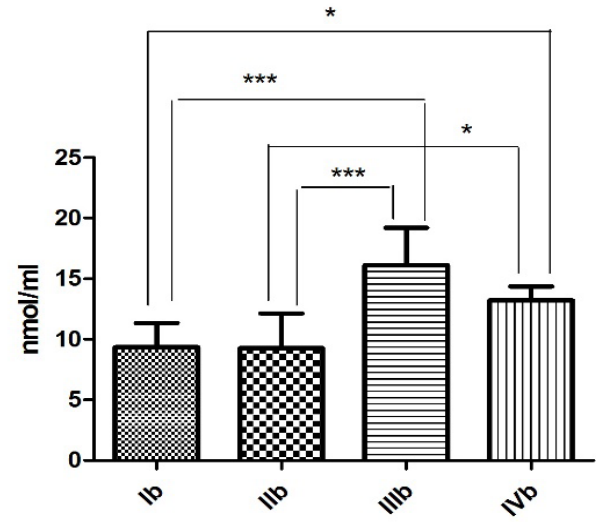

Figure 6. Glutathione levels in serum of animals pretreated with quercetin and curcumin 24 hours after carrageenan administration.

c) Quercetin and curcumin effects on oxidative stress parameters in lungs at $4 \mathrm{~h}$ after inflammation induction

In lung homogenates, malondialdehyde levels presented significant decreases in the rats that received quercetin (IVa, $\mathrm{p}<0.001$ ), in comparison with the control group (Ia) and carrageenan group (IIa) (Figure 7).

\section{Malondialdehyde in lung tissue - 4 hours}

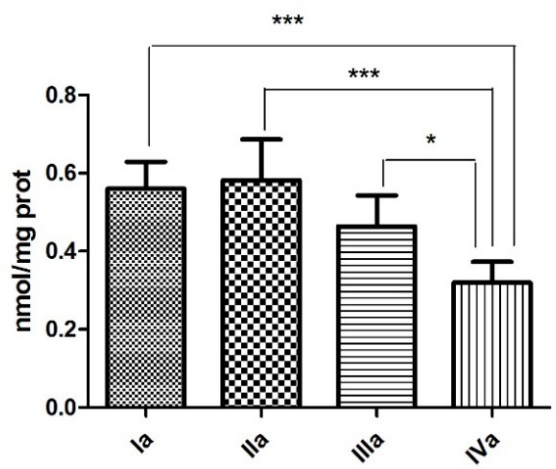

Figure 7. Malondialdehyde levels in the lung tissue of animals pretreated with quercetin and curcumin 4 hours after carrageenan administration.

At 4 hours after carrageenan administration, in lung homogenates, glutathione reduced levels did not present any significant variations among the groups (Figure 8). 
Glutathione in lung tissue - 4 hours

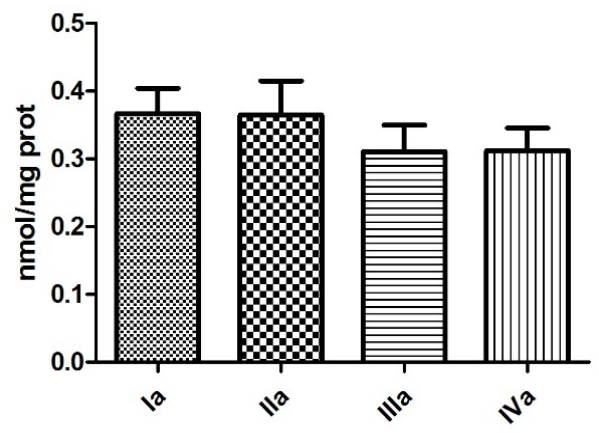

Figure 8. Glutathione levels in lung tissue of animals pretreated with quercetin and curcumin 4 hours after carrageenan administration.

d) Quercetin and curcumin effects on oxidative stress parameters in lungs at $24 \mathrm{~h}$ after inflammation induction

In the lungs, malondialdehyde levels did not have any significant variations among the groups (Figure 9).

Malondialdehyde in lung tissue - 24 hours

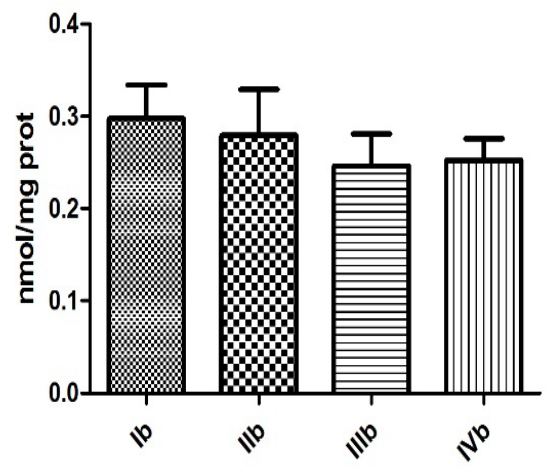

Figure 9. Malondialdehyde levels in the lung tissue of animals pretreated with quercetin and curcumin 24 hours after carrageenan administration.

At 24 hours after carrageenan administration, the levels of glutathione decreased significantly in the lungs of rats that were treated with antioxidants (groups IIIb and IVb, $\mathrm{p}<0.01$ ), in comparison with control (Ib) and carrageenan (IIb) groups (Figure 10).

\section{Glutathione in lung tissue - 24 hours}

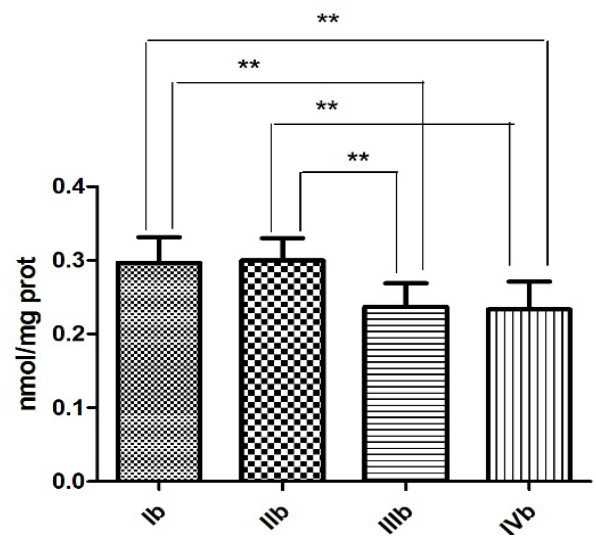

Figure 10. Glutathione levels in the lung tissue of animals pretreated with quercetin and curcumin at 24 hours after carrageenan administration.

\section{Discussion}

The results of our study demonstrated the redox imbalance in the serum and lungs, especially at 4 hours after intrapleural carrageenan administration. The rats that were not treated with antioxidants presented significantly high values of malondialdehyde, compared to the groups that received curcumin or quercetin prior to carrageenan injection.

The pro-oxidant effect of carrageenan was maintained 24 hours in the serum and in the lungs of the rats with pleural inflammation, evidenced by the significant increase of malondialdehyde generation in the rats without antioxidant protection, compared to the control group. It is known that carrageenan injection causes edema in rats in two phases, an initial phase up to 6 hours and a late phase extending up to 24 hours. In the early phase, histamine and serotonin are released, causing vasodilation while in the late phase, other chemicals become active: bradykinin, prostaglandins, neutrophil-derived ROS, nitric oxide, cytokines, proteases and lysososmal enzymes [12].

The mechanisms of the antioxidant defense were readily mobilised in the pre-treated rats with natural compounds: at 4 hours after carrageenan inflammation, the levels of ceruloplasmin and reduced glutathione were increased significantly in comparison with the untreated rats. After 24 hours, the antioxidant markers (ceruloplasmin and glutathione) were preserved at high levels in groups of rats that received curcumin and quercetin, compared to the groups without protection, suggesting the beneficial properties of the tested compounds.

In the lungs, at 4 hours after carrageenan administration, the oxidative stress was identified through significantly increased values of malondialdehyde in the rats without antioxidant administration, compared to the quercetin group. These significant modifications were not 
seen at 24 hours after the carrageenan inflammation. The glutathione levels in the lungs, at 24 hours after pleural inflammation, were reduced significantly in the rats that were previously treated with antioxidants, compared to the untreated groups, as an adaptive mechanism during the the oxidative agression.

Similar results were also found by Heeba et al. (2014) on a model of carrageenan-induced paw edema in rats. The same two antioxidants reduced the malondialdehyde and recovered the glutahione levels in the plantar tissue [13].

Curcumin and quercetin were analysed separately in different experimental models which simulated the lungs' pathology.

In 2013, Ma et al. induced acute inflammation of inferior respiratory pathways (asthma) in rats and demonstrated the favourable effect of curcumin [14]. The same properties were also observed by Shakeri and Boskabady (2017), suggesting that curcumin was implicated in the prevention of allergic inflammation in bronchial asthma [15]. Tenero et al. (2016) noticed in children with moderate to severe asthma the reduction of airway inflammation after the treatment with a combination of curcumin, resveratrol, soya phospholipids, zinc, selenium and vitamin D [16]. In 2018, Chauhan et al. evaluated the anti-inflammatory and antioxidant effects of sole curcumin and of combined curcumin with piperine on a mouse model of allergic asthma and they emphasized the therapeutic potential of intranasal administration of curcumin in the treatment of this disease [17].

In 2018, Zhu et al. demonstrated the curcumin protection in chronic obstructed pulmonary disease (COPD). Curcumin decreased the oxidative stress and the inflammation, induced by the cigarette smoke inhalation, through the signalling pathway PPAR $\gamma / \mathrm{NF}-\mathrm{\kappa B}$ in the human bronchial epithelial cells 16HBE [18]. In 2016 Tyagi et al. obtained favourable effects in reducing the inflammation and the oxidative stress through curcumin administration prior to the experimental paraquat exposure [19].

The curcumin effects were studied in vivo by Zang et al. (2015) on diabetes-induced pulmonary lesions, and the results demonstrated the inhibitory effects of this natural compound on NF- $\mathrm{KB}$ activation, oxidative stress and on inflammatory responses in the lungs [20].

Kumari et al. (2015) studied the effects of intranasal curcumin administration on lipopolysaccharide-induced acute lung inflammation, and observed the decrease of the oxidative stress parameters (nitric oxide, malondialdehyde), with the increase of the antioxidant protection (superoxide dismutase, catalase) [21].

Similar results were obtained by Huang et al. (2015) in their study performed on lipopolysaccharide-induced acute lung inflammation using quercetin: significant decreases of malondialdehyde level and significant increases of superoxide dismutase, catalase, and glutathione peroxidase in the lungs [22].
The protective effects of quercetin were studied in different in vitro and in vivo models of respiratory diseases. Taslidere et al. (2014) concluded that the treatment with quercetin in carbon tetrachloride-induced pulmonay injury may have positive effects in pulmonary diseases characterised by edema, inflammation and fibrosis [23]. Verma et al. (2013), in their study performed in a Wistar rat model of pulmonary fibrosis, presented the protective effect of quercetin on idiopatic lung fibrotic responses [24]. Veith et al. (2017) demonstrated also that quercetin reestablished the redox balance and reduced the inflammation in an in vitro model of idiopathic pulmonary fibrosis.

Quercetin increased the antioxidant response through the increase of Nrf2 activity and decreased, in a dosedependent manner, the production of pro-inflammatory cytokines [25]. Gardi et al. in 2015 have shown that quercetin attenuated all the inflammatory markers and oxidative stress parameters in a model of experimental arthritis. Quercetin reduced the levels of interleukin-1, C-reactive protein and monocyte chemo-attractant protein-1 and re-established the plasma antioxidant capacity. Additionally, quercetin inhibited the enzymatic activity of pro-inflammatory 12/15-lipoxygenase in lungs and liver, and increased the heme oxigenase- 1 expression in the joints and in the lungs of the arthritic rats [26]. In 2006, Jackson et al. performed a comparative analyse of curcumin and quercetin in an arthritis experimental model and observed the superior effects of curcumin [27].

To the best of our knowledge, no similar experimental models were performed, but our results are concordant with the research made on other models of pleural and lung inflammation.

In our study, the administration of both natural compounds prior to carrageenan administration presented antioxidant actions, beneficial effects that could be considered as an alternative adjuvant treatment of lung diseases with inflammatory processes.

\section{Conclusions}

In serum, at 4 and at 24 hours, curcumin and quercetin had protective effects, reduced the oxidative stress and stimulated the antioxidant defence. Curcumin had superior antioxidant effects to quercetin, probably because of a better bioavailability and absorbtion. In lung homogenates, at 4 hours, only quercetin reduced significantly the lipid peroxidation, a beneficial effect that was not recorded at 24 hours.

Our results showed that the oral administration of curcumin or quercetin could improve the antioxidant protection during the lung inflammatory disseases.

\section{Acknowledgements}

The authors would like to thank Nicoleta Decea and Remus Moldovan from the Laboratory of Research in the 
Oxidative Stress, Physiology Department, Iuliu Haţieganu University of Medicine and Pharmacy, for their help in the experimental work.

\section{References}

1. Middleton E Jr, Kandaswami C, Theoharides TC. The effects of plant flavonoids on mammalian cells: implications for inflammations, heart disease, and cancer. Pharmacol Rev. 2000;52:673-751.

2. Bartholomé R, Haenen G, Hollman CH, Bast A, Dagnelie PC, Roos D, et al. Deconjugation kinetics of glucuronidated phase II flavonoid metabolites by beta-glucuronidase from neutrophils. Drug Metab Pharmacokinet. 2010;25:379-387.

3. Bischoff SC. Quercetin: potentials in the prevention and therapy of disease. Curr Opin Clin Nutr Metab Care. 2008;11:733-740.

4. Boots AW, Haenen GR, Bast A. Health effects of quercetin: from antioxidant to nutraceutical. Eur J Pharmacol. 2008;585:325-337.

5. Toda S, Miyase T, Arichi H, Tanizawa H, Takino Y. Natural antioxidants. III. Antioxidative components isolated from rhizome of Curcuma longa L. Chem Pharm Bull (Tokyo). 1985;33:1725-1728.doi: 10.1248/cpb.33.1725.

6. Jurenka JS. Anti-inflammatory properties of curcumin, a major constituent of Curcuma longa: a review of preclinical and clinical research. Altern Med Rev. 2009;14:141-153.

7. Dzoyem JP, McGaw LJ, Kuete V, Bakowsky U. Therapeutic potential against metabolic, inflammatory, infectious and systemic diseases. In: Kuete V (editor). Medicinal Spices and Vegetables from Africa. Elsevier Inc., Academic Press, 2017; p. 239-270.

8. Petronilho F, de Souza B, Vuolo F, Benetton CA, Streck EL, Roesler R, et al. Protective effect of gastrin-releasing peptide receptor antagonist in carrageenan-induced pleural inflammation in rats. Inflamm Res. 2010;59:783-789.

9. Conti M, Morand PC, Levillain P, Lemonnier A. Improved fluorometric determination of malonaldehyde. Clin Chem. 1991;37:1273-1275.

10. Hu ML. Measurement of protein thiol groups and glutathione in plasma. Methods Enzymol. 1994;233:380-384.

11. Ravin HA. An improved colorimetric enzymatic assay of ceruloplasmin. J Lab Clin Med. 1961;58:161-168.

12. Posadas I, Bucci M, Roviezzo F, Rossi A, Parente L, Sautebin L, et al. Carrageenan-induced mouse paw oedema is biphasic, age-weight dependent and displays differential nitric oxide cyclooxygenase-2 expression. Br J Pharmacol. 2004; 142:331-338.

13. Heeba GH, Mahmoud ME, El Hanafy AA. Anti-inflammatory potential of curcumin and quercetin in rats: role of oxidative stress, heme oxygenase- 1 and TNF- $\alpha$. Toxicol Ind Health. 2014;30:551-560.
14. Ma C, Ma Z, Fu Q, Ma S. Curcumin attenuates allergic airway inflammation by regulation of $\mathrm{CD} 4+\mathrm{CD} 25+$ regulatory $\mathrm{T}$ cells (Tregs)/Th17 balance in ovalbumin-sensitized mice. Fitoterapia. 2013;87:57-64.

15. Shakeri F, Boskabady MH. Anti-inflammatory, antioxidant, and immunomodulatory effects of curcumin in ovalbuminsensitized rat. Biofactors. 2017;43:567-576.

16. Tenero L, Piazza M, Zanoni L, Bodini A, Peroni D, Piacentini GL. Antioxidant supplementation and exhaled nitric oxide in children with asthma. Allergy Asthma Proc. 2016;37:e8-e13.

17. Chauhan PS, Jaiswal A, Subhashini, Singh R. Combination therapy with curcumin alone plus piperine ameliorates ovalbumin-induced chronic asthma in mice. Inflammation. 2018;41:1922-1933.

18. Zhu T, Shi C, Li H, He J, Yang Y, Wang Q, et al. Curcumin suppresses cigarette smoke extract-induced oxidative stress through PPAR $\gamma / \mathrm{NF}-\kappa \mathrm{B}$ pathway in human bronchial epithelial cells in vitro. Nan Fang Yi Ke Da Xue Xue Bao. 2018;38:1209-1214.

19. Tyagi N, Dash D, Singh R. Curcumin inhibits paraquat induced lung inflammation and fibrosis by extracellular matrix modifications in mouse model. Inflammopharmacology. 2016;24:335-345.

20. Zhang F, Yang F, Zhao H, An Y. Curcumin alleviates lung injury in diabetic rats by inhibiting nuclear factor- $\kappa \mathrm{B}$ pathway. Clin Exp Pharmacol Physiol. 2015;42:956-963.

21. Kumari A, Tyagi N, Dash D, Singh R. Intranasal curcumin ameliorates lipopolysaccharide-induced acute lung injury in mice. Inflammation. 2015;38:1103-1112.

22. Huang $\mathrm{R}$, Zhong $\mathrm{T}, \mathrm{Wu} \mathrm{H}$. Quercetin protects against lipopolysaccharide-induced acute lung injury in rats through suppression of inflammation and oxidative stress. Arch Med Sci. 2015;11:427-432.

23. Taslidere E, Esrefoglu M, Elbe H, Cetin A, Ates B. Protective effects of melatonin and quercetin on experimental lung injury induced by carbon tetrachloride in rats. Exp Lung Res. 2014;40:59-65.

24. Verma R, Kushwah L, Gohel D, Patel M, Marvania T, Balakrishnan S. Evaluating the ameliorative potential of quercetin against the bleomycin-induced pulmonary fibrosis in Wistar rats. Pulm Med. 2013;2013:921724.

25. Veith C, Drent M, Bast A, van Schooten FJ, Boots AW. The disturbed redox-balance in pulmonary fibrosis is modulated by the plant flavonoid quercetin. Toxicol Appl Pharmacol. 2017;336:40-48.

26. Gardi C, Bauerova K, Stringa B, Kuncirova V, Slovak L, Ponist $\mathrm{S}$, et al. Quercetin reduced inflammation and increased antioxidant defense in rat adjuvant arthritis. Arch Biochem Biophys. 2015;583:150-157.

27. Jackson JK, Higo T, Hunter WL, Burt HM. The antioxidants curcumin and quercetin inhibit inflammatory processes associated with arthritis. Inflamm Res. 2006;55:168-175. 